\title{
Equidecomposability of Polyhedra with Reference to Crystallographic Groups
}

\author{
Carsten Müller \\ Sektion Mathematik, Friedrich-Schiller-Universität, UHH, Jena 6900 , \\ German Democratic Republic
}

Communicated by $\mathrm{J}$. Wills

\begin{abstract}
This paper deals with the introduction of theoretical statements which are the results of studying equidecomposability of polyhedra with reference to discrete transformation groups. Lattice polygons and paving polyhedra play the most important role. The equivalence of volume equality and equidecomposability of any two of this polyhedra is shown for special discrete groups.
\end{abstract}

\section{Introduction}

We consider spaces $(R, G)$, where $R$ is the usual $d$-dimensional Euclidian space $E^{d}$ and $G$ is a crystallographic group of $E^{d}$. So $G$ contains $d$ linearly independent translation vectors generating the subgroup $V$ of translations. The investigation of those spaces is motivated by models such as lattice point spaces, scanning fields, or other ideas relevant to practice.

The crystallographic groups can be described by an orbit of a point $x$ of $E^{d}$. The orbit $G(x)$ is the set of all points $x^{\prime}$ for which there exists a transformation $g^{\prime}$ of $G$ where $g^{\prime}(x)=x^{\prime}$. We select the point $x$ so that the number of transformations $g$ of $G$ where $g(x)=x$ is maximal. So it is clear that all lattices which describe $G$ are equivalent under the group of all transformations.

We speak of $G$-equality of two sets of points $A$ and $B$ if there exists a transformation $g$ of $G$ such that $g(A)=B$. A generalization of this relation leads to the important concept of the $G$-equidecomposability. Two sets of points $A$ and $B$ are called $G$-equidecomposable if there are dissections of $A$ and $B$ in a finite number of pairwise $G$-equal subsets.

The concept of the fundamental domain is essential for the work with crystallographic groups. This is a closed set $T$ of points of $E^{d}$, where at least one point 
of each orbit lies in $T$ but no two $G$-equal points lie in the interior of $T$. It is possible to select the fundamental domain as a compact set, because the subgroup of translations is $d$-dimensional.

\section{Foundations}

The substantial ideas, which are connected with $G$-equidecomposability of polyhedra, come from Hadwiger (see [6] and [7]). He defines the degree of belonging $\omega(x, A)$ of a point $x$ of $E^{d}$ to a polyhedron $A \subset E^{d}$. That means the proportion between the volume of the intersection of $K(x, \varepsilon)$ with the polyhedron $A$ and the volume of $K(x, \varepsilon)$. Here $K(x, \varepsilon)$ is a ball with its centre in $x$ and a sufficiently small radius $\varepsilon$. So the value $\varphi_{x}(A)$ is defined for every point $x$ of $E^{d}$ and any polyhedron $A$ to be the product of $v_{x}$ with the sum of all values $\omega\left(x^{\prime}, A\right)$, where $x^{\prime}$ is an element of $G(x)$ and $v_{x}$ is the number of transformations of $G$ where $g(x)=x$ (Fig. 1).

The equality of $\varphi_{x}(A)$ and $\varphi_{x}(B)$ for all $x$ of $E^{d}$ follows from the $G$ equidecomposability of two polyhedra $A$ and $B$ because this functional $\varphi_{x}$ is additive and $G$-invariant. Hadwiger also proves the converse of this fact.

Theorem 1. If $A$ and $B$ are polyhedra of $E^{d}, G$ is a crystallographic group of $E^{d}$ and if, for all points $x$ of $E^{d}, \varphi_{x}(A)=\varphi_{x}(B)$ holds then $A$ and $B$ are G-equidecomposable.

The proof is constructive. The analysis of the proof allows an algorithm which determines the $G$-equidecomposability of two polyhedra (of equal volume) in a finite number of steps.

If we consider only pure discrete translation groups $V$ with $d$ linearly independent vectors, Hadwiger proves that any translates of lattice parallelotops $P$ and $Q$ of equal volume are always $V$-equidecomposable (see Fig. 2). Here a lattice

$$
\left.W_{3}=\{a, b\} \text { (3) [p 3 }\right]
$$

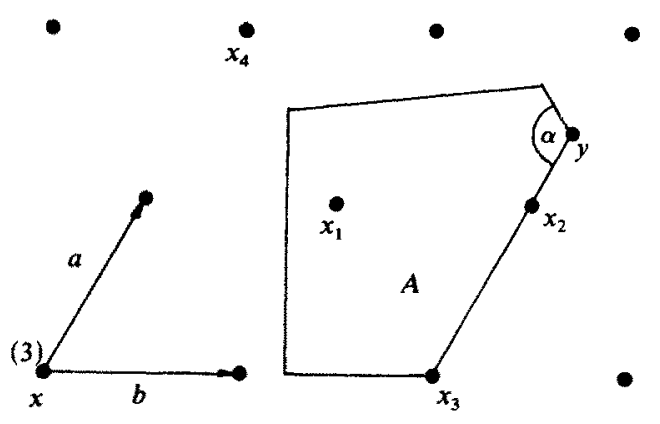

$$
\begin{aligned}
\omega\left(x_{1}, A\right) & =1 \\
\omega\left(x_{2}, A\right) & =0.5 \\
\omega\left(x_{3}, A\right) & =0 . \overline{3} \\
\omega\left(x_{4}, A\right) & =0 \\
\omega(y, A) & =\frac{\alpha}{2 \pi} \\
\varphi_{x}(A) & =3(1+0.5+0 . \overline{3})=5.5
\end{aligned}
$$

Fig. 1 


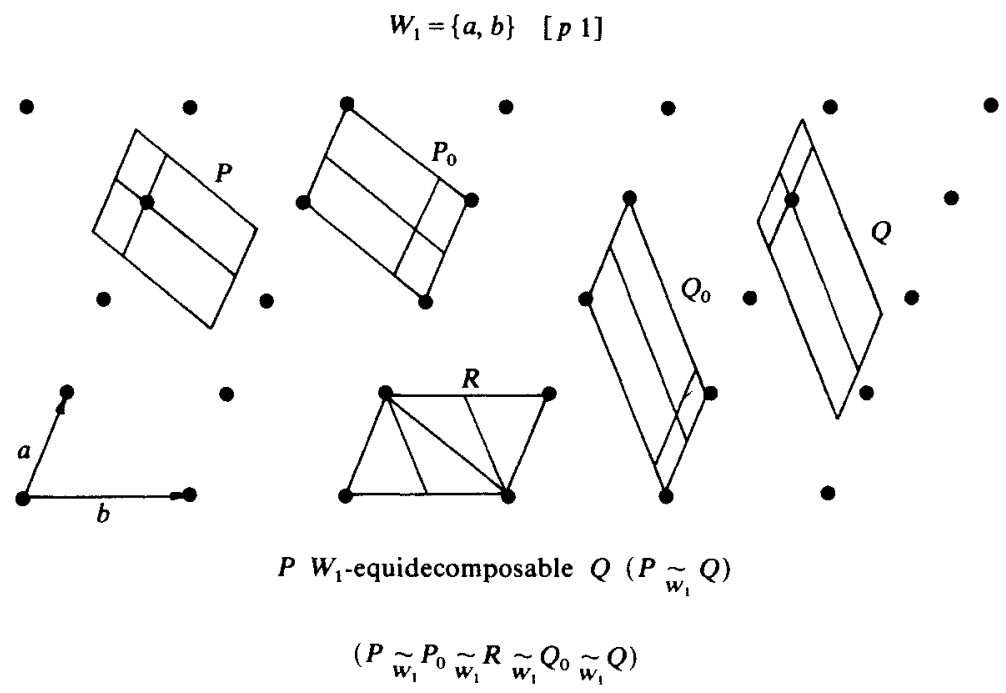

Fig. 2

polyhedron is a polyhedron whose vertices belong to the lattice describing the group $G$.

\section{Lattice Polygons}

As was seen there are remarkable statements about $G$-equidecomposability, although the possibilities of transformations of $G$ are very much restricted. Lattice polyhedra play an important role. The $G$-equidecomposability of lattice polyhedra has very nice parallels with the equidecomposability of common polyhedra, as in the classical transformation groups. It is well known that two polygons of equal area in the plane are always $S$-equidecomposable where $S$ is the group of all translations and all point reflections in the plane. The counterpart to this classical result is given in

Theorem 2. Two lattice polygons $A$ and $B$ of equal area are always $W_{2}$-equidecomposable where $W_{2}$ has two noncollinear vectors $a$ and $b$ and one point reflection as generators.

Proof. It is possible to dissect the lattice polygons $A$ and $B$ into lattice triangles as in the classical situation. We can dissect these lattice triangles into (so-called) simplest lattice triangles. Simplest lattice triangles are lattice triangles which have no other lattice points on the edges or in the interior. It is easy to see that the area of every two simplest lattice triangles are equal. It follows that the number of such simplest triangles, after the dissection of $A$ and $B$, is equal independent of the concrete dissection. So it is sufficient for proof of Theorem 2 to prove the $W_{2}$-equidecomposability of two simplest lattice triangles. 


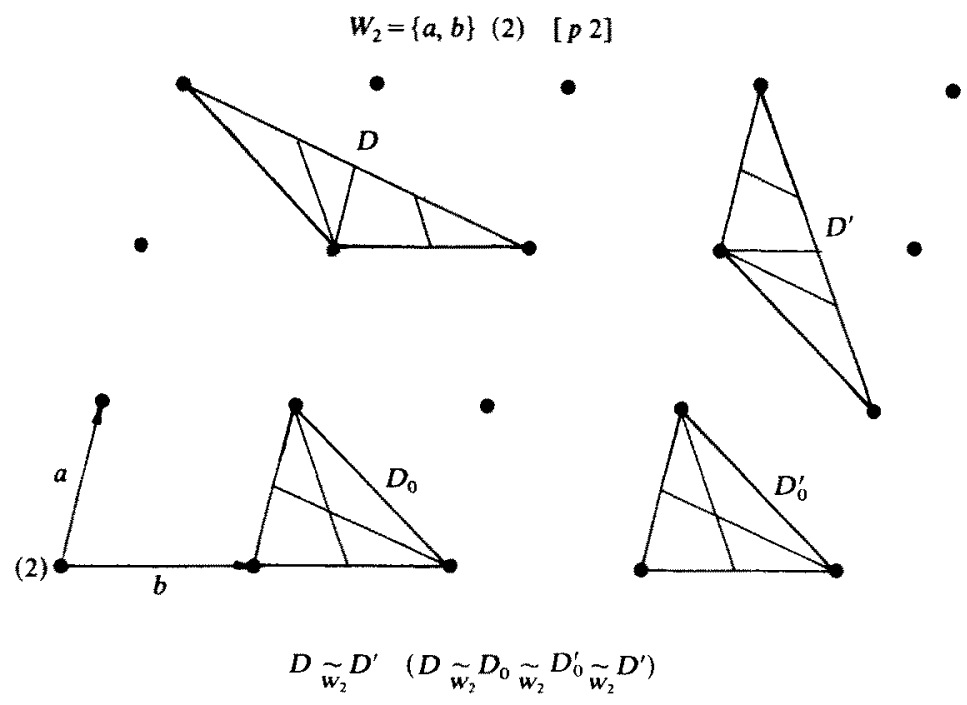

Fig. 3

Simplest lattice triangles under the group $W_{2}$ are paving polyhedra (see definition of paving polyhedra at the beginning of Section 4). It follows that Theorem 2 is a corollary of Theorem 3 .

An example is given in Fig. 3.

The transitivity of the $G$-equidecomposability in connection with the statements at the end of Section 2 allows us to conclude that any translated lattice parallelogram is $W_{2}$-equidecomposable to any lattice polygon, if they are of equal area. The lattice polygon can even be translated by a vector $n(a / 2)+m(b / 2)$ with the integers $n$ and $m$.

It is not possible to make the same statement of Theorem 2 in the threedimensional case. There exist lattice polyhedra of equal volume which are not $G$-equidecomposable by the lattice describing group $G$. This is parallel to the Third Hilbert Problem.

Finally, we have reason to believe that other concrete results are possible in this direction. The proof exists, for instance, in the case of squares of equal area with at least two nonneighboring vertices of order 4 which are always $W_{4}$ equidecomposable.

Betke and Kneser [1] studied other $F$ groups and the consequences to the $F$-equidecomposability and the $F$-equicompletability of lattice polyhedra in the lattice of integers $Z^{d}$. It is surprising that in their case $F$-equicompletability and $F$-equidecomposability are not equivalent. In this case the equality of volumes is equivalent to $F$-equicompletability for lattice polyhedra. $G$-equicompletability and $G$-equidecomposability by crystallographic groups $G$ are always equivalent, because the existence of the functional $\varphi_{x}$ guarantees the correctness of the Subtraction Theorem. 


\section{Paving Polyhedra}

There exists another set of polyhedra with the property that the equality of volumes is equivalent to the $G$-equidecomposability if $G$ is a crystallographic group. In this section a polyhedron $P$ will be a $G$-paving polyhedron in the following sense:

1. There exists a subgroup $G^{\prime}$ of $G$ such that the union of all $g^{\prime}(P)$ is the space $E^{d}$ where $g^{\prime}$ is an element of $G^{\prime}$.

2. The intersection of $g_{1}(P)$ and $g_{2}(P)$ has no interior points, if $g_{1}$ and $g_{2}$ are two different transformations of $G^{\prime}$.

It seems that it is not a natural requirement that $G^{\prime}$ should be a subgroup of $G$. There are other common subsets $G^{\prime \prime}$ of $G$ that can also realize the paving. It is, on the one hand, sensible to require this for the proof of Theorem 3 and, on the other hand, it is conjectured that for any convex $G$-paving polyhedron with subset $G^{\prime \prime}$ of $G$ there also exists a subgroup $G^{\prime}$ of $G$ which realizes a possibly different paving with the same polyhedron.

The assertion of this section can now be given in

Theorem 3. Two G-paving polyhedra $P_{1}$ and $P_{2}$ ( $G$ being a crystallographic group) are $G$-equidecomposable if they are of equal volume.

Proof. Since $P_{i}$ are paving polyhedra there exist subgroups $G_{i}(i=1,2)$ of $G$ and it is clear that the polyhedra $P_{i}$ are also fundamental domains of $G_{i}(i=1,2)$. It is simple to prove that two fundamental domains $T^{\prime}$ and $T^{\prime \prime}$ of the same crystallographic group $G^{\prime}$ are $G^{\prime}$-equidecomposable. For this we assume that $T^{\prime}$ and $T^{\prime \prime}$ are simultaneous paving polyhedra of $G^{\prime}$.

We will now construct two fundamental domains $T_{1}$ and $T_{2}$ of $G_{1}$ and $G_{2}$ which are $G$-equidecomposable. Thus the dissection of $G$ into cosets is used. The indexes of $G_{1}$ and $G_{2}$ are finite because the polyhedra $P_{1}$ and $P_{2}$ are bounded. We now assume $G=\bigcup_{k=1}^{j_{i}} a_{k}^{i} G_{i}\left(a_{k}^{i} \in G ; i=1,2\right)$. We will show that the sets $T_{i}=\bigcup_{k=1}^{j_{1}} a_{k}^{i}(T)$ are fundamental domains of $G_{i}(i=1,2)$. Therefore it is necessary to prove the properties of fundamental domains for $T_{i}$ which are formulated at the end of Section 1.

1. The choice of the elements $a_{k}^{i}$ guarantees that the union of $g_{i}\left(T_{i}\right)$ over all $g_{i} \in G_{i}(i=1,2)$ fills the entire space $E^{d}$. Thus the orbit of an arbitrary point $x$ of $E^{d}$ lies in the union $\bigcup_{g_{1} \in G_{i}} g_{i}\left(T_{i}\right)(i=1,2)$. It follows that $x$ is an element of a subset $g_{i_{0}}\left(T_{i}\right)$. So the point $g_{i_{0}}^{-1}(x)$ is an element of $T_{i}(i=1,2)$. This means that at least one point of every orbit lies in $T_{i}(i=1,2)$.

2. The choice of the $a_{k}^{i}\left(k=1, \ldots, j_{i} ; i=1,2\right)$ can be made in such a way that $a_{k_{1}}^{i}(T) \cap a_{k_{2}}^{i}(T)$ is empty as long as $k_{1} \neq k_{2}$. This is because the number of cosets of $G$, with respect to $G_{1}$ and $G_{2}$, is finite, and also because there is a $d$-dimensional subgroup of translations in $G$. Thus the interior points of $T_{i}(i=1,2)$ may be simultaneous interior points of $T$. The assumption that two $G_{i}$-equivalent points lie in the interior of $T_{i}(i=1,2)$ obviously leads to a contradiction in the properties of the fundamental domain $T$ of $G$. 


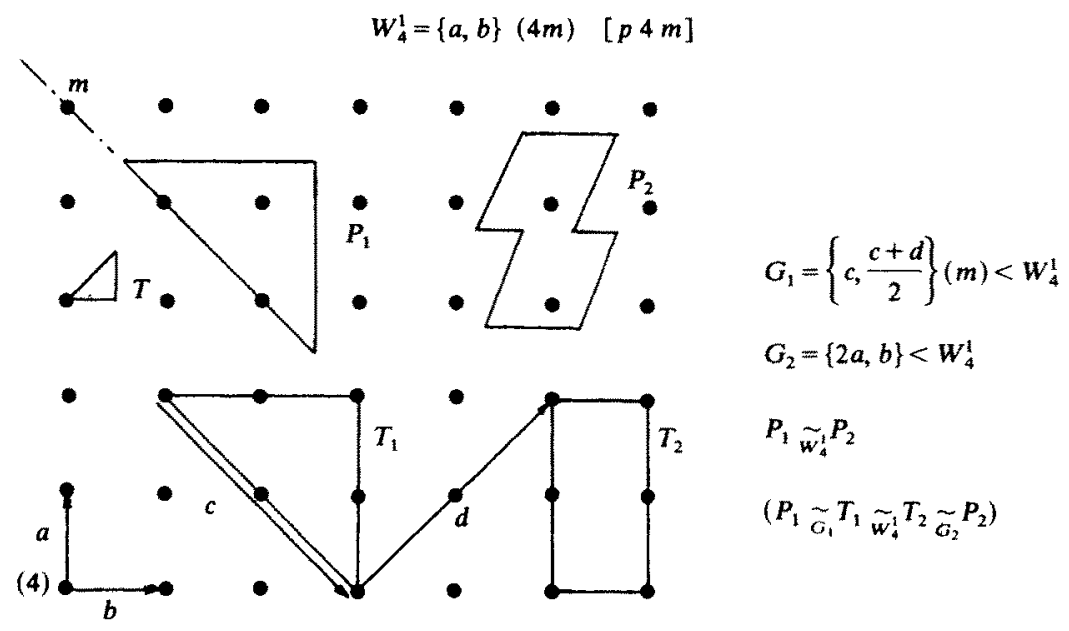

Fig. 4

It is clear that the intersection of the two different sets $a_{r}^{i}(T)$ and $a_{s}^{i}(T)$ $\left(1 \leq r, s \leq j_{i} ; i=1,2\right)$ has no interior points. Hence the polyhedra $T_{1}$ and $T_{2}$ are $G$-equidecomposable, since the $P_{i}(i=1,2)$ are of equal volume, and so it equally holds that $j_{1}=j_{2}$. Transitivity and symmetry of $G$-equidecomposability complete the proof and establish the theorem (see also Fig. 4).

The result of Theorem 3 can be compared with investigations of similar questions by Debrunner in [3] and [4]. The difference is that our study of crystallographic groups is more limited. On the other hand, Theorem 3 holds for arbitrary paving polyhedra and does not confine itself only to concrete types of polyhedra.

As Theorem 3 holds for all finite dimensions $d$ of $E^{d}$ it is possible to formulate a special conclusion:

Two nonequidecomposable polyhedra of equal volume in $E^{3}$ cannot be G-paving polyhedra in one and the same crystallographic group $G$.

\section{References}

1. U. Betke and M. Kneser, Zerlegungen und Bewertung von Gitterpolytopen, J. Reine und Angew. Math. 358 (1985), 202-208.

2. L. Danzer, B. Grünbaum, and G. C. Shepard, Does every type of polyhedron tile three-space? Structural Topology 8 (1983), 3-14.

3. H. E. Debrunner, Pflasterung des euklidischen Raumes mit kongruenten Simplexen, Bern, 1978, Manuscript.

4. H. E. Debrunner, Über Zerlegungsgleichheit von Plasterpolyedern mit Würfeln, Arch. Math. (Basel) 35 (1980), 583-587. 
5. B. Grünbaum and G. C. Shepard, Tiling with congruent tiles, Bull. Amer. Math. Soc. 3 (1980), $951-973$.

6. H. Hadwiger, Uber Gitter und Polyeder, Monatsh. Math. 57 (1953), 246-254,

7. H. Hadwiger, Vorlesungen über Inhalt, Oberfläche und Isoperimetrie, Springer-Verlag, Berlin, Göttingen, Heidelberg, 1957.

8. C. Müller, Zerlegungsgleichheit von Punktmengen bezüglich diskreter Transformationsgruppen, Dissertation, Friedrich-Schiller-Universität, Jena, 1986.

Received August 14, 1986, and in revised form January 28, 1987. 